\title{
A new algorithm for water retention on magic square
}

\author{
Shatha A. Salman, Amal A.Ibrahim \\ Mathematics and Computer Application, Applied Sciences, University of Technology, Iraq
}

\begin{tabular}{l}
\hline Article Info \\
\hline Article history: \\
Received Apr 20, 2020 \\
Revised Apr 21, 2020 \\
Accepted May 5, 2020 \\
\hline
\end{tabular}

Keywords:

Algorithm

Hetero magic square

Magic square

Ultra- magic square

Water retention

Corresponding Author:

Shatha Assaad Salman, Department of Applied Sciences, University of Technology,

Al-Sinaa Street, Baghadad, Iraq.

Email:100178@uotechnology.edu.iq

\section{INTRODUCTION}

Magic squares being a mathematical theme of entertainment attracted the attention of many mathematicians and therefore magic squares are considered an example of the beauty of mathematics subjects and have many applications [1-6]. The first magic square is found in China and is called Lo Shu square which is a square matrix with positive integers in which all the lines (rows, column, diagonals) are equal to the same value. The magic squares attracted wide attention, which led to their transition to India, Europe and the Islamic world in order to study them and to know their characteristics. The prevailing belief then was that these squares possessed magical properties and therefore were linked in many cultures by astrology and divination [7-9]. In recent years magic squares have been linked with water retention to reach the magic squares that trap as much as possible of water. The problem of water retention is to pour water on a certain surface and calculate the amount of water that will settle inside the holes of that surface. The rest of the excess water is leaking through the edges, and thus finding maximum magic square that retaining water. Figure 1 illustrates a magic square whose height is determined by the value within each cell.

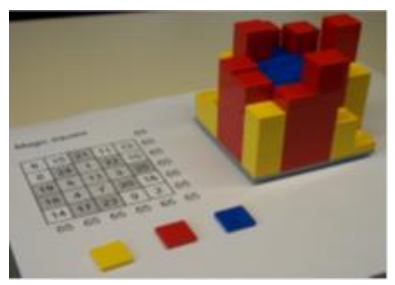

Figure 1. Magic square representation 


\section{WATER RETENTION}

Due to the importance of water in many aspects of life, in addition to climate change, it was necessary to find ways to store water and one of these methods are storage of water on the mathematical surfaces. Water retention on magic squares is one of the beautiful applications of magic square where the water is stored in the magic square by generating a wall surrounds the cells with the smallest values in which the retention of water. It was reported for the first time in 2007 by Knecht. In 2010 a competition was organized to find the magic squares which can store as much water as possible. It is possible to determine the total units of water trapped by finding the difference between each cell within the wall with the lowest value of the wall and collect all the resulting values to find the total value. The principle of storing water on magic square does not depend on the natural magic squares, but overrides it to other types of magic squares, for example semi-magic square, pandiagonal magic square, associative magic square and ultra-magic square as shown in Figure 2. For more details about this application see [10-16]. Other applications are also used magic square in combinatorics [17-21]

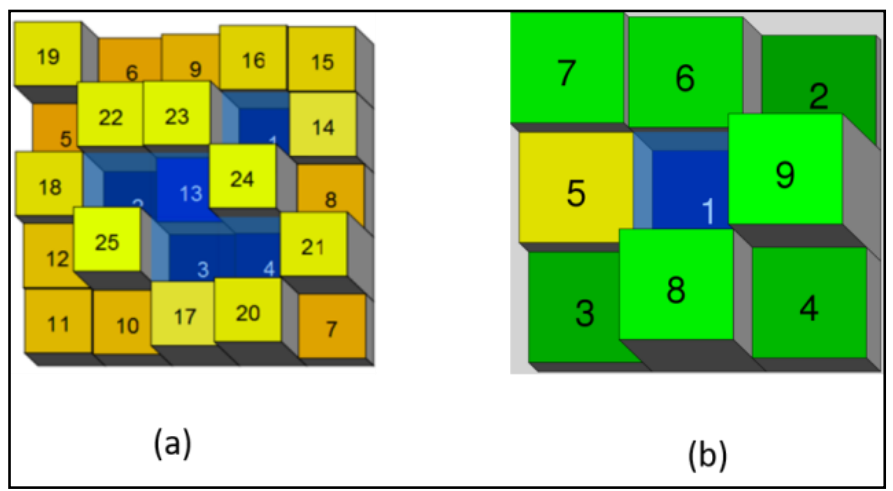

Figure 2. (a) Associative magic square retaining 56 units of water (b) Semi -magic square retaining 4 units of water

\section{SOME TYPES OF MAGIC SQUARE}

In this section some definitions and types for the magic square are given.

\section{Definition (1).[22]}

Semi-magic square is a magic square which satisfies the condition of magic except that the sum of one of the diagonals or both is not equal to the magic constant as given in Figure 3.

\begin{tabular}{|l|l|l|}
\hline 1 & 5 & 9 \\
\hline 6 & 7 & 2 \\
\hline 8 & 3 & 4 \\
\hline
\end{tabular}

Figure 3. Semi magic square

Definition (2). [23]

In a square matrix which achieved the condition of a natural magic square and in addition the sum of the broken diagonals are equal to the magic constant it is called a pandigonal magic square which is given in Figure 4.

\begin{tabular}{|c|c|c|c|c|}
\hline 1 & 15 & 24 & 8 & 17 \\
\hline 23 & 7 & 16 & 5 & 14 \\
\hline 20 & 4 & 13 & 22 & 6 \\
\hline 12 & 21 & 10 & 19 & 3 \\
\hline 9 & 18 & 2 & 11 & 25 \\
\hline
\end{tabular}

Figure 4. Pandigonal magic square 
Definition (3). [24]

A natural magic square for which each pair of numbers is symmetric versus centre, sum to $n^{2}+1$ is known as associative magic square as in Figure 5.

\begin{tabular}{|l|l|l|}
\hline 4 & 9 & 2 \\
\hline 3 & 5 & 7 \\
\hline 8 & 1 & 6 \\
\hline
\end{tabular}

Figure 5. Associative magic square

Definition (4).

Squares that are both pan diagonal and associative are called ultra-magic as given in Figure 6.

\begin{tabular}{|c|c|c|c|c|c|c|}
\hline 27 & 46 & 31 & 1 & 6 & 17 & 47 \\
\hline 8 & 9 & 20 & 26 & 34 & 43 & 35 \\
\hline 29 & 28 & 11 & 32 & 37 & 36 & 2 \\
\hline 45 & 38 & 40 & 25 & 10 & 12 & 5 \\
\hline 48 & 14 & 13 & 18 & 39 & 22 & 21 \\
\hline 15 & 7 & 16 & 24 & 30 & 41 & 42 \\
\hline 3 & 33 & 44 & 49 & 19 & 4 & 23 \\
\hline
\end{tabular}

Figure 6. Ultra-magic square

Definition (5). [25]

Hetero square is square matrix whose entries positive numbers from 1 to $n^{2}$ such that the sum of rows, columns, and diagonals has different sums. It can be constructed by arranging successive integers in a spiral way as in Figure 7.

\begin{tabular}{|l|l|l|}
\hline 9 & 8 & 7 \\
\hline 2 & 1 & 6 \\
\hline 3 & 4 & 5 \\
\hline
\end{tabular}

Figure7. Hetero square

\section{AN ALGORITHM FOR BUILDING WATER RETENTION ON MAGIC SQUARE OF ORDER $7,9,11,13$.}

Two algorithms are given in this section for magic squares of order 7, 9, 11 and 13

\section{Algorithm 1}

Input: magic square of order $n$

Output: the unit of water retention on magic square.

Step 1

The first and last rows and the first and last columns are a wall surrounding the inner matrix.

Step 2

The corners of the inner matrix can be determined using the following four formulas.

$\mathrm{a}=\max \left(J_{n-5, m-5}, \min \left(J_{n-6, m-5}, J_{n-5, m-6}\right)\right)$

$\mathrm{b}=\max \left(J_{n-1, m-5}, \min \left(J_{n-1, m-6}, J_{n, m-5}\right)\right)$

$\mathrm{c}=\max \left(J_{n-5, m-1}, \min \left(J_{n-6, m-1}, J_{n-5, m}\right)\right)$

$\mathrm{d}=\max \left(J_{n-1, m-1}, \min \left(J_{n, m-1}, J_{n-1, m}\right)\right)$ 


\title{
Step 3
}

The corners of the row that divide the internal matrix into two equal parts can be found using the following formulae.

$$
\begin{aligned}
& \mathrm{e}=\max \left(J_{n-3, m-5}, \min \left(J_{n-3, m-6}, \mathrm{a}, \mathrm{b}\right)\right) \\
& \mathrm{f}=\max \left(J_{n-3, m-1}, \min \left(J_{n-3, m}, \mathrm{c}, \mathrm{d}\right)\right) \\
& \text { Step } 4
\end{aligned}
$$

The corners of the column that divides the internal matrix into two equal parts can be determined using the following formulas.

$$
\begin{aligned}
& \mathrm{g}=\max \left(J_{n-5, m-3}, \min \left(J_{n-6, m-3}, \mathrm{a}, \mathrm{c}\right)\right) \\
& \mathrm{h}=\max \left(J_{n-1, m-3}, \min \left(J_{n, m-3}, \mathrm{a}, \mathrm{b}\right)\right)
\end{aligned}
$$

Step 5

Find the pillars of the second column of the inner matrix as follows.

$$
\begin{aligned}
& \left.\mathrm{i}=\max J_{n-5, m-4}, \min \left(J_{n-6, m-4}, \mathrm{a}, \mathrm{b}\right)\right) \\
& \left.\mathrm{j}=\max J_{n-1, m-4}, \min \left(J_{n, m-4}, \mathrm{~b}, \mathrm{~d}\right)\right)
\end{aligned}
$$

Step 6

Find the pillars of the fourth column of the inner matrix as follows.

$\mathrm{k}=\max \left(J_{n-5, m-2}, \min \left(J_{n-6, m-2}, \mathrm{a}, \mathrm{b}\right)\right)$

$1=\max \left(J_{n-1, m-2}, \min \left(J_{n, m-2}, \mathrm{a}, \mathrm{c}\right)\right)$

Step 7

Find the pillars of the second row of the inner matrix as follows.

$\mathrm{m}=\max \left(J_{n-4, m-5}, \min \left(J_{n-4, m-6}, \mathrm{c}, \mathrm{d}\right)\right)$

$\mathrm{n}=\max \left(J_{n-4, m-1}, \min \left(J_{n-4, m}, \mathrm{c}, \mathrm{d}\right)\right)$

Step 8

Find the pillars of the fourth row of the inner matrix as follows.

$\mathrm{O}=\max \left(J_{n-2, m-5}, \min \left(J_{n-2, m-6}, \mathrm{~b}, \mathrm{~d}\right)\right)$

$\mathrm{P}=\max \left(J_{n-2, m-1}, \min \left(J_{n-2, m}, \mathrm{~b}, \mathrm{~d}\right)\right)$

Step 9

For the cells that lie in the centre of the inner matrix there are two steps.

1. For the main diagonal and all diagonals parallel to it, find the greatest element with the exception of values that were previously extracted

2. The same procedure is followed for the second diagonal and the parallel diagonal for it.

By using the formula $\mathrm{z}=\max \left(J_{n-3, m-3}, \min (\mathrm{g}, \mathrm{h}, \mathrm{e}, \mathrm{f})\right)$ Which varies according to the selected cell, where $J_{n-3, m-3}$ is the cell located in the center and $(\mathrm{g}, \mathrm{h}, \mathrm{e}, \mathrm{f})$ are the corners of the row and column that the intersection of them is the cell $j_{n-3, m-3}$.

\section{Step 10}

If $J_{n-5, m-5}$ is less than $J_{n-6, m-5}$ then consider $J_{n-6, m-5}$ to be a block in the wall and if $J_{n-5, m-5}$ is less than $J_{n-5, m-6}$ then consider $J_{n-5, m-6}$ as a block in the wall. Otherwise if $J_{n-5, m-5}$ is less than the both then they both cell are a blocks in the wall. If $J_{n-5, m-5}$ is greater than both cell this mean that it is a block in the wall.

\section{Step 11}

If $J_{n-1, m-5}$ is less than $J_{n-1, m-6}$ then consider $J_{n-1, m-6}$ to be a block in the wall and if $J_{n-1, m-5}$ is less than $J_{n, m-5}$ then consider $J_{n, m-5}$ as a block in the wall. Otherwise if $J_{n-1, m-5}$ is less than both cells then they are a blocks in the wall. If $J_{n-1, m-5}$ is greater than both cells this mean that it is a block in the wall.

\section{Step 12}

If $J_{n-5, m-1}$ is less than $J_{n-6, m-1}$ then consider $J_{n-6, m-1}$ to be a block in the wall and if $J_{n-5, m-1}$ is less than $J_{n-5, m}$ then consider $J_{n-5, m}$ as a block in the wall. Otherwise if $J_{n-5, m-1}$ is less than both cells then they are a blocks in the wall. If $J_{n-5, m-1}$ is greater than both cells this mean that it is a block in the wall.

\section{Step 13}

If $J_{n-1, m-1}$ is less than $J_{n-1, m}$ then consider $J_{n-1, m}$ to be a block in the wall and if $J_{n-1, m-1}$ is less than $J_{n, m-1}$ then consider $J_{n, m-1}$ as a block in the wall. Otherwise if $J_{n-1, m-1}$ is less than both cells then they are blocks in the wall. If $J_{n-1, m-1}$ is greater than both cells this mean that it is a block in the wall.

\section{Step 14}

The amount of water that are retained in the magic square are calculated by finding the difference between every cell that retaining water and the smallest value in the wall, at least calculate the sum of them.

\author{
Algorithm 2 \\ Step 1 \\ Consider the first and last rows and the first and last columns as a wall surrounding the inner matrix.
}


Step 2

Divide the matrix into four parts as shown.

\begin{tabular}{|l|l|l|}
\hline$A$ & & $C$ \\
\hline & & \\
\hline$B$ & & $D$ \\
\hline
\end{tabular}

\section{Step 3}

The cells of the Wall that are located in part A can be determined using the same method as in the following formula and the coordinates change depending on the location of the selected cell.

$\mathrm{A}=\max \left(J_{22}, \min \left(J_{12}-J_{21}\right)\right.$

Step 4

The cells in part B are determined using the same way as in the following formula.

$\mathrm{B}=\max \left(J_{62}, \min \left(J_{61}-J_{72}\right)\right.$

Step 5

The cells in $\mathrm{C}$ are determined as in the formula.

$\mathrm{C}=\max \left(J_{26}, \min \left(J_{16}-J_{27}\right)\right.$

Step 6

To find the cells in D use the same method as in the formula.

$\mathrm{D}=\max \left(J_{66}, \min \left(J_{76}-J_{67}\right)\right.$

Step 7

If $J_{22}$ is less than $J_{12}$ then consider $J_{12}$ as a block in the wall and if $J_{22}$ is less than $J_{21}$ then consider $J_{21}$ to be a block in the wall otherwise if $J_{22}$ is less than both cells then they are blocks in the wall if $J_{22}$ is greater than both cells, this mean that it is a block in the wall.

\section{Step 8}

If $J_{62}$ is less than $J_{61}$ then consider $J_{61}$ as a block in the wall and if $J_{62}$ is less than $J_{72}$ then consider $J_{72}$ to be a block in the wall. Otherwise if $J_{62}$ is less than both cells then both cells are blocks in the wall if $J_{62}$ is greater than both cells this mean that it is a block in the wall.

\section{Step 9}

If $J_{26}$ is less than $J_{61}$ then consider $J_{16}$ to be a block in the wall and if $J_{26}$ is less than $J_{27}$ then consider $J_{27}$ as a block in the wall. Otherwise if $J_{26}$ is less than both cells then both cells are blocks in the wall if $J_{26}$ is greater than both cell this mean that it is a block in the wall.

\section{Step 10}

If $J_{66}$ is less than $J_{67}$ then consider $J_{67}$ as a block in the wall and if $J_{67}$ is less than $J_{76}$ then consider $J_{76}$ to be a block in the wall. Otherwise if $J_{66}$ is less than both then both cells are blocks in the wall if $J_{66}$ is greater than both cells this mean that it is a block in the wall.

The last four steps are only executed for the coordinates listed.

\section{EXAMPLES}

Some examples of water retention algorithms using magic squares for order 5, 7 and 9. Now an example for Applying algorithm 1 is introduce.

Example (1): To find the units of water retention on the magic square of order 7.

Step 1

Consider the first and last rows and the first and last columns as a wall surrounding the inner matrix.

\begin{tabular}{|l|l|l|l|l|l|l|}
\hline 4 & 25 & 46 & 19 & 31 & 32 & 18 \\
\hline 27 & 44 & 16 & 45 & 12 & 2 & 29 \\
\hline 47 & 10 & 14 & 6 & 48 & 20 & 30 \\
\hline 21 & 43 & 1 & 42 & 5 & 41 & 22 \\
\hline 33 & 11 & 49 & 3 & 24 & 15 & 40 \\
\hline 34 & 7 & 13 & 37 & 17 & 39 & 28 \\
\hline 9 & 35 & 36 & 23 & 38 & 26 & 8 \\
\hline
\end{tabular}




\section{Step 2}

$\mathrm{a}=\max (44, \min (25,27))=44$

$\mathrm{b}=\max (7, \min (34,35))=34$

$\mathrm{c}=\max (2, \min (32,29))=29$

$\mathrm{d}=\max (39, \min (28,26))=39$

Step 3

$\mathrm{e}=\max (43, \min (21,44,34))=43$

$\mathrm{f}=\max (41, \min (22,29,39))=41$

Step 4

$\mathrm{g}=\max (45, \min (19,44,29))=45$

$\mathrm{h}=\max (37, \min (23,44,34))=37$

Step 5

$\mathrm{i}=\max (16, \min (46,44,29))=29$

$\mathrm{j}=\max (13, \min (36,34,39))=34$

Step 6

$\mathrm{k}=\max (12, \min (31,44,34))=31$

$1=\max (17, \min (38,44,29))=29$

Step 7

$\mathrm{m}=\max (10, \min (47,29,39))=29$

$\mathrm{n}=\max (20, \min (30,29,38))=29$

Step 8

$\mathrm{o}=\max (11, \min (33,34,39))=33$

$\mathrm{p}=\max (15, \min (40,34,39))=34$.

Step 9

$q=\max (42, \min (45,37,43,41))=42$.

$\mathrm{r}=\max (48, \min (31,29,29,29))=48$.

$\mathrm{s}=\max (49, \min (29,34,33,34))=49$.

\section{Step 10}

$44>25$ and 27 , thus 44 is block in the wall.

Step 11

$7<34$ and 36, thus 36 and 34 are blocks in the wall.

Step 12

$2<32$ and 29, thus 32 and 29 are blocks in the wall.

Step 13

$39>28$ and 26, thus 39 is block in the wall.

\section{Step 14}

$(29-12)+(29-2)+(29-20)=53$

$(42-16)+(42-10)+(42-14)+(42-6)+(42-1)=163$

$(33-11)+(33-7)+(33-13)=68$

$(37-5)+(37-3)+(37-24)+(37-15)+(37-17)=121$

Units $=53+163+68+121=405$ in Figure 8 .

\begin{tabular}{|l|l|l|l|l|l|l|}
\hline & 5 & 6 & 9 & 1 & 2 & 8 \\
\hline 7 & 4 & 6 & 5 & 2 & & 9 \\
\hline 7 & 0 & 4 & & 8 & 0 & 0 \\
\hline 1 & 3 & & 2 & & 1 & 2 \\
\hline 3 & 1 & 9 & & 4 & 5 & 0 \\
\hline 4 & & 3 & 7 & 7 & 9 & 8 \\
\hline & 5 & 6 & 3 & 8 & 6 & \\
\hline
\end{tabular}

Figure 8. Magic square of order 7 with 405 units of retention 
Now an example for applying algorithm 2 is introduce.

Example (2): To find the units of water retention on the magic square of order 5, the following steps are used Step 1

The wall of the matrix is the $1^{\text {st }}$ and $5^{\text {th }}$ row and the $1^{\text {st }}$ and $5^{\text {th }}$ column.

Step 2

Divide the matrix into four parts as shown.

\begin{tabular}{|l|l|l|l|l|}
\hline & 1 & 2 & 0 & \\
\hline 4 & 4 & & & 1 \\
\hline 5 & & 3 & & 9 \\
\hline 8 & & & 5 & \\
\hline 0 & 7 & 6 & 3 & \\
\hline
\end{tabular}

Step 3: find the value in block A

$a_{1}=\max (24, \min (11,14))$

$a_{2}=\max (1, \min (22,24))$

$a_{3}=\max (6, \min (24,15))$

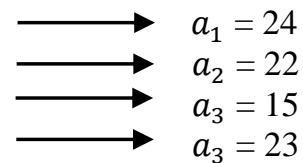

$a_{4}=\max (23, \min (1,6))$

Step 4: find the value in block B

$b_{1}=\max (7, \min (18,17))$

lock C

$c_{1}=\max (5, \min (20,21))$

D

Step 6: find the value in block $D$

$d_{1}=\max (25, \min (12,13))$

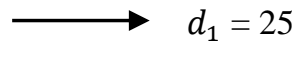

\begin{tabular}{|l|l|l|l|l|}
\hline & 1 & 2 & 0 & \\
\hline 4 & 4 & & & 1 \\
\hline 5 & & 3 & & 9 \\
\hline 8 & & & 5 & 2 \\
\hline 0 & 7 & 6 & 3 & \\
\hline
\end{tabular}

The wall cells that are calculated using the algorithm.

The block from the wall that surround the inner matrix.

The cell which retains water.

Calculate the unit of retaining water as follow

The lowest value in the wall is 15

$(15-1)+(15-5)+(15-2)+(15-6)+(15-7)+(15-3)$

The unit of the cell that retaining water is 66 units

\section{CONCLUSION}

Magic square is very important in many real life applications; our work gives one of their important applications which are water retention. New algorithms are written for magic square of order 7 then the work was expanded to include all odd magic square. 


\section{REFERENCES}

[1] A.A.Abiyev, et al., "Investigation of center of mass by using magic squares and its possible engineering applications," Robotics and Autonomous systems, vol. 49, no. (3-4), pp. 219-226, 2004

[2] L.H.Cox, "Bounds on entries in 3-dimensional contingency tables subject to given marginal totals," Inference Control in Statistical Databases - From Theory to Practice. Lec. Not. Comp. Sci. Springer, New York, series 2316, pp. 21-33, 2002.

[3] A. S. Shatha,"Computation of odd magic square using a new approach with some properties," Eng. \& Tech. Journal, vol. 30, no. 7, pp.1203-1210, 2012.

[4] A. S. Shatha, "Some properties of magic squares of distinct squares and cube magic squares," Al-Mustansiriyah Journal of Science, vol. 30, no. 3,pp. 60-63, 2019.

[5] D. X. Zeng, et al, "Overview of rubik's cube and reflections on its application in mechanism," Chinese Journal of Mechanical Engineering, 31, no.1, pp.77, 2018.

[6] N.O.Schmidt, "Latin squares and their applications to cryptography," MS.C. Boise State University, 2016.

[7] D.L.Anderson, "Magic squares: discovering their history and their magic," Mathematics Teaching in the Middle School, 6, no. 8, pp.466, 2001.

[8] B. L. Kaul and R. Singh, "Generalization of magic square (numerical logic) $3 \times 3$ and its multiples $(3 \times 3) \times(3 \times$ 3)," IJ Intelligent Systems and Applications, vol. 1, pp. 90-97, 2013.

[9] J.Sesiano, "Magic squares: Their history and construction from ancient times to AD 1600," Springer, 2019.

[10] S. H. Jamel, et al., "Steps for constructing magic cube using two orthogonal Latin squares and a magic square," In Proceeding of MUCEET Malaysian Technical Universities Conference on Engineering and Technology, pp. 20-22, 2009.

[11] Nordgren, R. P., "On properties of special magic square matrices," Linear Algebra and its Applications, vol.437, no. 8, pp. 2009-2025, 2012.

[12] Öfverstedt, J., "Water retention on magic squares with constraint-based local search," Uppsala University, 2012.

[13] D. B. Ojha, et al., "Generalization of 4× 4 magic square," International Journal of Applied Engineering Research, vol. 1, no. 4, pp.706, 2010.

[14] M. Trenkler, "Connections-magic squares, cubes and matchings," Applications of Modern Mathematical Methods, Ljubljana, 191199, 2001.

[15] Jr, T. Trotter, "Normal magic triangles of order n," Journal. of Recreational Mathematics, vol. 5, no.1, pp. 28-32, 1972

[16] T. R. Hagedorn, "Magic rectangles revisited," Discrete mathematics, vol. 207, no.1-3, pp. 65-72, 1999.

[17] J.A.De Loera, Kim, E.D., Onn, S. and Santos, F., "Graphs of transportation polytopes," Journal of Combinatorial Theory, Series A, vol. 116, no.8, pp. 1306-1325, 2009.

[18] C. A. Floudas, "Deterministic global optimization: theory, methods and applications," Springer Science \& Business Media, vol. 37, 2013.

[19] J. A. Lindon, "Anti-magic squares," Mathematics, vol. 7, pp.16-19, 1962

[20] T. S. Motzkin, "The multi-index transportation problem," Bull. Amer. Math. Soc, vol. 58, no. 4, pp. 494, 1952.

[21] L. J. Mordell, "On sums of three cubes," Journal of the London Mathematical Society, vol 1, no. 3, pp.139-144, 1942.

[22] M. M. Ahmed, "Algebraic combinatorics of magic square," Ph.D. thesis, University of California, 2004.

[23] W.S.Andrews, "Magic squares and cubes," Cosimo, Inc., 2004.

[24] B.Aronov, et al., "A generalization of magic squares with applications to digital halftoning," Theory of Computing Systems, vol. 42, no. 2, pp.143-156, 2008.

[25] J. S. Madachy, "Madachy's mathematical recreations", Dover Publications, 1979.

\section{BIOGRAPHIES OF AUTHORS}

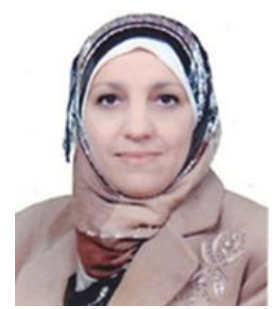

Shatha Salman is Ph.D. degree from Al-Nahrain University in 2005. She has become assistant professor on November 2005. Her Master of Applied Mathematics from University of Technology Iraq (1995). She obtained Bachelor Degree in Applied Mathematics from University of Technology (Iraq) in 1990. Her researches are in fields of Geometric Combintorics, Abstract Algebra, Graph theory, Differential Equations Ordinary and Fractional, Numerical Analysis and Complex Analysis. As well, she has been a member of Iraqi of Scientific committee since 1995. She has served as invited reviewer. She has 40 published articles inside Iraq and some in international journals, 2 published books in Germany. Further info on his scholargoogle. https://scholar.google.com/citations?user=_WbhHyQAAAAJ\&hl=en\&authuser=1

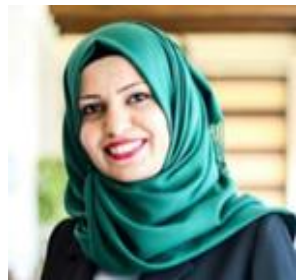

Amal A. Ibrahim Graduate of the University of Technology, Department of Applied Sciences, Mathematics Branch, Computer Applications 2015,She obtained a master's degree from the Department of Applied Sciences, Mathematics Branch of Computer Applications 2019. Her researches are in fields of Geometric Combintorics, Abstract Algebra, Graph theory; She has some published articles inside Iraq and some in international journals. 